HNO 2009 $\cdot 57: 417-420$

DOI 10.1007/s00106-009-1906-3

Online publiziert: 25. April 2009

(c) Springer Medizin Verlag 2009

\author{
H.-W. Pau \\ Universitäts-Hals-Nasen-Ohren-Klinik, Rostock
}

\title{
Die Geburtsstunde der HNO-Heilkunde schlug in Rostock
}

für das hervorragende internationale Referenten gewonnen werden konnten.

Die sich abzeichnende Größe der Veranstaltung mit ihren erneut über 700 Beiträgen in Form von Vorträgen, Multimediapräsentationen, Postern sowie zahlreichen Hauptvorträgen, „invited lectures" und Rundtischgesprächen ließ alle vor Ort verfügbaren Kongressräume als zu klein erscheinen, sodass wir es erstmals gewagt haben, in der architektonisch ansprechenden ehemaligen großen Halle der Internationalen Gartenbauausstellung, die heute als Messehalle dient, „maßgeschneiderte“ Vortragsräume herrichten zu lassen. Das Ambiente der Halle, ihre Lage innerhalb von Grünflächen und die Nähe zum Meer mit dem Ostseebad Warnemünde mögen gute Voraussetzungen für einen wissenschaftlichen Gedankenaustausch gewährleisten!

\section{( Technik für Lebensqualität - Biomaterialien und Implantate in der HNO-Heilkunde}

Das Motto des diesjährigen Jahreskongresses heißt: „Technik für Lebensqualität - Biomaterialien und Implantate in der Hals-Nasen-Ohren-Heilkunde“. Mit diesem Thema befassen sich 11 Referate, für die jeweils besonders ausgewiesene Experten gewonnen werden konnten. Eine kurze Einführung in diese Problematik soll später in diesem Editorial gegeben werden. Über diese Referatethemen hinaus werden auf unserem Treffen Schwerpunkte wie Funktionsstörungen der Tuba auditiva, M. Menière, Auswirkungen von Reflux im HNO-Bereich, Innovati- onen auf dem Gebiet der Bildgebung, Anwendung von Botulinumtoxin, neue Entwicklungen bei der Tonsillektomie, Konzepte zur Tinnitustherapie und Aktuelles zur Ballon-Sinuplastik in Rundtischgesprächen diskutiert. Besondere Bedeutung haben die aktuellen Entwicklungen der Onkologie, für die sogar 2 Rundtischdiskussionen vorgesehen sind: zum einen $\mathrm{zu}$ neuen therapeutischen Konzepten, zum anderen zu der für unser Fach sehr wichtigen Entwicklung neuer Organisationsstrukturen.

Am „Tag der Praxis“ werden Probleme des betagten Patienten sowie Möglichkeiten ambulanter Lasertherapie im Vordergrund stehen, während das Hauptthema des Pflegetages der tracheotomierte Patient sein wird.

Das auf der letzten Jahrestagung in Bonn eingeführte und bewährte Prinzip frühmorgendlicher „state of the art lectures" in englischer Sprache wird beibehalten, auch hierfür konnten hervorragende Experten gewonnen werden.

Die Fülle des Programms lässt eine vollständige Auflistung sämtlicher Schwerpunkte nicht $\mathrm{zu}$, stellvertretend seien hier noch die Orbitachirurgie als interdisziplinäre Aufgabe zu sowie aktuelle Entwicklungen zum Kochleaimplantat $(\mathrm{CI})$ genannt.

\section{Implantate und Biomaterialien}

Implantate und Biomaterialien spielen auch in der Hals-Nasen-Ohren-Heilkunde eine große Rolle. Man versteht darunter in den Körper eingepflanzte Materialien, die dauernd oder zumindest über
Kongress in jeweils einem Auditorium ein

Programm in englischer Sprache geboten, 


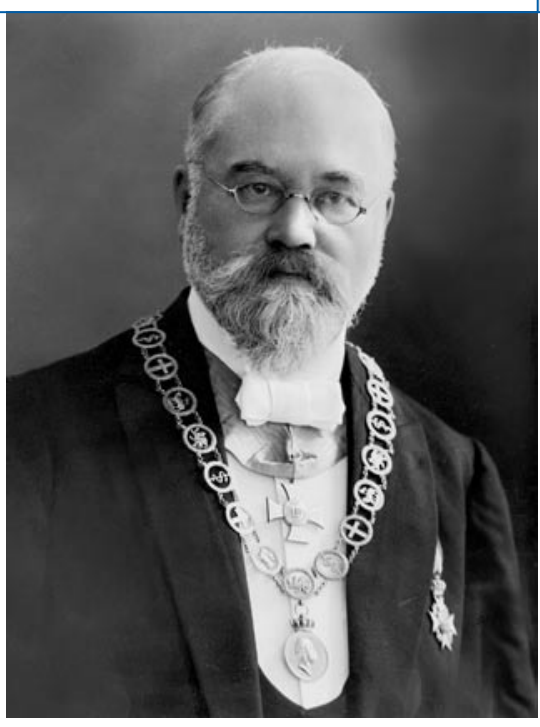

Abb. $1 \Delta$ Prof. Otto Körner, erster Lehrstuhlinhaber für unser gesamtes Fachgebiet

eine längere Zeitspanne verbleiben sollen. Für die Referate wurden dazu die Begriffe bewusst sehr weit gefasst, sodass auch eher äußerliche Hilfsmittel wie z. B. Trachealkanülen oder Nasentamponaden in die Betrachtung eingeschlossen wurden. Die Beschäftigung mit Biomaterialien ist nicht neu, verlangt aber unbedingt einen interdisziplinären Ansatz mit enger $\mathrm{Zu}$ sammenarbeit zwischen Medizin, Naturund Ingenieurwissenschaft. Solche Ansätze werden in den letzten Jahren zunehmend beispielsweise durch die DFG gefördert, wobei durchaus auch überregionale Kooperationen entstehen, wie z. B. der Transregio-Sonderforschungsbereich 37, an dem Universitäten der Standorte Aachen, Hannover und Rostock beteiligt sind. Auch für die zunehmend in den Vordergrund tretende „regenerative Medizin“ oder das Tissue-Engineering sind Biomaterialien unverzichtbare Bestandteile. Die den Kongress eröffnenden Referate sollen dabei den aktuellen Stand der Entwicklung von Implantaten und Biomaterialien für unser Fachgebiet darstellen.

Dazu ist es wichtig, die Anforderungen an diese künstlichen Ersatzmaterialien und ihre Funktionen zu definieren. So macht es große Unterschiede, ob das Material nur zur Überbrückung eines temporären Funktionsausfalls dienen und den Körper unter Resorption des Fremdstoffes zur Selbstheilung stimulieren soll oder ob ein permanenter Defekt durch ein „künstliches Organ“ wie z. B. ein implantierbares Hörgerät oder ein Kochleaim- plantat behoben werden soll. Im Übrigen weisen alle Referate darauf hin, dass es das (ideale) Biomaterial oder das Implantat für alle Fälle nicht gibt, sondern dass man allenfalls auf dem Wege ist, für bestimmte Anforderungen die Materialien zu optimieren.

Dazu dient auch die Bemühung, Oberflächen schon funktionierender Implantatsysteme zu „funktionialisieren“, sodass beispielsweise Wachstumsfaktoren oder infektionshemmende Substanzen über bestimmte zeitliche Intervalle abgegeben werden.

\section{Otologie}

Kochleaimplantate werden seit über 20 Jahren als Prothesen bei taub geborenen Kindern oder postlingual ertaubten Erwachsenen höchst erfolgreich eingesetzt. Trotz dieser Erfolgsgeschichte gibt es weiterhin hohen Verbesserungsbedarf, insbesondere vor dem Hintergrund, dass der CI-Träger lebenslang auf diese Prothese angewiesen ist. Dabei ist die Bruchsicherheit, elektrische Zuverlässigkeit sowie Dichtigkeit des Systems genauso zu nennen wie die Notwendigkeit, Keiminvasionen ins Innenohr oder die Bildung von oberflächlichen Biofilmen zu verhindern - schließlich verläuft ja das Kabel frei durch das nicht sterile Mittelohr. Auch intrakochleäre Reaktionen auf den Elektrodenträger sowie unerwünschter Zellbewuchs der Elektroden mit Verschlechterung der elektrischen Leitfähigkeit sind $\mathrm{zu}$ beachten. In einem Referat werden aktuelle Entwicklungen wie die Einsatzmöglichkeiten neuer Materialien oder die Funktionalisierung von Oberflächen z. B. zur lokalen Freisetzung von Pharmaka ausführlich erläutert.

Teilweise ähnliche Anforderungen gelten für Mittelohrimplantate, sei es, dass sie als passive Implantate nur Defekte der Gehörknöchelchenkette überbrücken oder als aktive Implantate zusätzlich Vibrationen über die Rest-Ossikelkette oder über die Fenster direkt ans Innenohr bringen. Aus einer Vielzahl bislang verwendeter Materialien hat sich v. a. das Titan als besonders geeignet herausgestellt. Zur Ankopplung an noch funktionierende Reste der Gehörknöchelchen wurden filigrane
Clip-Mechanismen entwickelt und erfolgreich eingesetzt.

Grundvoraussetzung des Funktionierens ist jedoch eine intakte Mittelohrventilation über die Tube. Schon allein ein mangelnder Druckausgleich mit Einziehung des Trommelfells kann den Schalltransport erheblich verschlechtern, was noch stärker für die Bildung von Retraktionstaschen oder Atelektasen gilt. Ist nur eine Prothese zwischen Trommelfell und Fußplatte interponiert („total ossicular replacement prosthesis“, TORP) so können auch atmosphärische Druckschwankungen durch diese Columella ohne Zwischenschaltung von Gelenken auf die Fußplatte übertragen werden. Auch wenn hier eine direkte Gefährdung des Innenohrs unwahrscheinlich ist, erscheint doch die Integration eines Gelenks in den Prothesenschaft sinnvoll.

Die Entwicklung aktiver, vibrationsinduzierender Prothesen ist in vollem Gange, wobei durch eine zunächst unkonventionelle Applikation einer Vibrant Soundbridge (angekoppelt an die Rundfenstermembran) ihr Wert auch für die Versorgung von Schallleitungsschwerhörigkeiten deutlich wurde. Neben dieser „Rundfensterankopplung“ werden derzeit neue Möglichkeiten klinisch und experimentell gesucht, das Innenohr durch Vibrationen zu stimulieren.

\section{Schädelknochen und Schädelbasis}

Biomaterialien zur Wiederherstellung des knöchernen Schädels werden zur Osteosynthese nach Frakturen bzw. zur Rekonstruktion knöcherner Defekte eingesetzt.

Zur Osteosynthese werden meist Minioder Mikroplatten aus Titanlegierungen eingesetzt, die hinsichtlich Biokompatibilität, Stabilität sowie Anpassungsmöglichkeit an das Implantatbett überzeugen. Nach wie vor wird die Frage ihrer Entfernungsnotwendigkeit nach abgeschlossener Frakturheilung kontrovers diskutiert. Eine solche entfällt natürlich bei resorbierbaren Osteosynthese-Systemen, die sich bei vergleichbarer mechanischer Stabilität v. a. für den kindlichen Schädel eignen. Knochenersatzmaterialien werden in großer Anzahl entwickelt und angeboten, ohne dass bislang eine ideale, alle Anforderung erfüllende Substanz gefunden 
wurde. Derzeitige Entwicklungen versuchen, eine körpereigene Knochenneubildung anzuregen, etwa über eine Strukturierung der Implantatoberfläche oder den Einsatz z. B. von „bone morphogenetic protein“ (BMP). Die Verwendung durch Tissue-Engineering hergestellten Knochens ist bislang noch Zukunftsmusik.

Zunehmende Bedeutung, auch für die Rekonstruktion der Schädelbasis, erlangt der Einsatz präoperativ CT-gestützt geplanter und patientenspezifisch präformierter Titan- oder Keramikimplantate. Dabei werden jedoch auch weiterhin intraoperativ modellierbare formende $\mathrm{Ti}$ tannetze zur Rekonstruktion der Schädelbasis bzw. der Orbita Anwendung finden.

Bei Defekten der Dura sind - sofern ohne Zusatzgefährdung des Patienten verfügbar - autogene Materialien (Galeaperiost, Schleimhautperichondrium, Fascia lata, Temporalisfaszie) bevorzugt einzusetzen, während bei allo- oder xenogenen Geweben (meist Kollagen) trotz sehr hoher Sicherheitsstandards ein infektiöses Restrisiko nicht völlig ausgeräumt werden kann. Alloplastische Materialien haben diese Probleme nicht, v. a. resorbierbare Substanzen erscheinen in vieler Hinsicht vorteilhaft. Auch könnte der zusätzliche Einsatz von Wachstumsfaktoren Fortschritte bringen.

\section{Oberer Aerodigestivtrakt}

Im Gegensatz zu den guten Ergebnissen des Einsatzes von Implantaten und Biomaterialien zum Knochen- oder Duraersatz sind Defekte des schleimhautausgekleideten oberen Aerodigestivtrakts bis heute kaum durch Fremdmaterialien zu schließen. Versuche mit unterschiedlichsten Materialien zur Trachealrekonstruktion haben bislang nicht zu einer erfolgreichen klinischen Anwendung geführt, da Probleme wie Nahtinsuffizienz, Stenosierung, Störung der Blutversorgung sowie ausbleibende mukoziliäre Clearance auftraten. Es bleibt abzuwarten, ob der derzeitige Ansatz über das Tissue-Engineering neue therapeutischen Optionen für die rekonstruktive Trachealchirurgie eröffnen wird.

Das Gleiche gilt für die Pharynxrekonstruktion, bei der sich Biomaterialien

Abb. $2>$ Erste deutsche Klinik für Hals-, Nasen- und Ohrenheilkunde in Rostock

(1899)

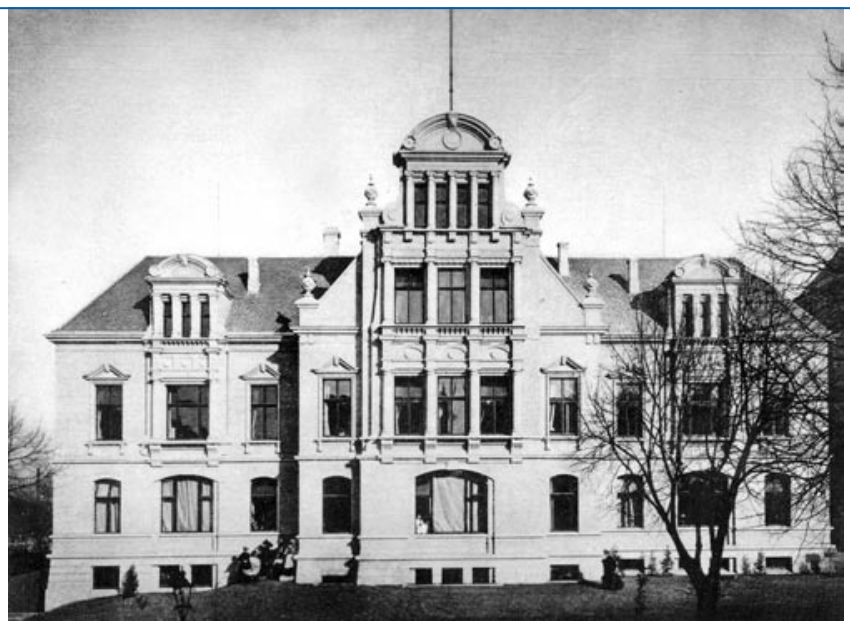

Abb. $3 \triangleright$ Heutige Otto-Körner-Klinik für

Hals-, Nasen- und

Ohrenheilkunde in Rostock

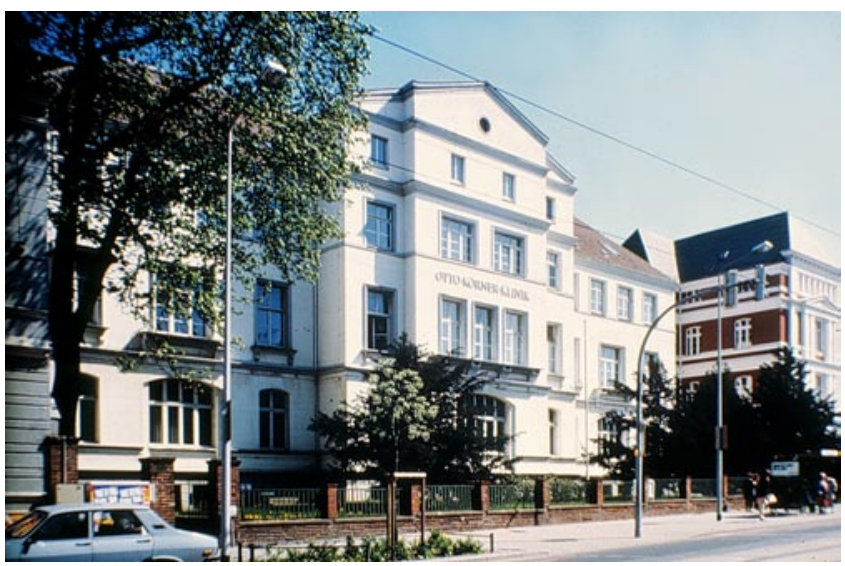

in einem Experimentalstadium befinden, das im Referat näher erläutert wird. Auch hier werden für die Zukunft große Erwartungen in das Tissue-Engineering gesetzt.

Im Bereich des Kehlkopfes werden Implantate eingesetzt, um durch Medialisierung einer gelähmten, lateral stehenden Stimmlippe wieder einen zur Phonation geeigneten Glottisschluss zu ermöglichen. Dazu dienen zum einen feste, von außen durch den gefensterten Schildknorpel in den paraglottischen Raum eingebrachte Implantate, zum anderen aber auch injizierbare Materialien wie Polydimethylsiloxan, Hydroxylapatit, Kollagen oder Hyaluronsäure. In Gegensatz zu den beiden erstgenannten Substanzen werden Kollagen und Hyaluronsäure im zeitlichen Verlauf wieder abgebaut, sodass ihr Medialisierungseffekt eher temporär ist („Probefahrt“). Die verschiedenen soliden Implantate aus unterschiedlichen Materialien haben meist vergleichbare Resultate hinsichtlich des Medialisierungseffekts bzw. der Stimm- bildung, Unterschiede bestehen eher im operativen Handling.

Laryngeale Stents dienen zur Schienung des Kehlkopfgerüsts in frühen postoperativen Phasen, sie müssen individuell angepasst werden.

Auch für Trachealkanülen und Hilfsmittel zur Pflege eines Tracheostomas steht heute ein großes Angebot zur Verfügung, das in einem eigenen Referat dargestellt wird. Für den jeweiligen Patienten muss bezüglich Material, Art und Form der Kanüle sowie zusätzlicher Hilfsmittel (z. B. "heat and moisture exchanger", HME) eine möglichst optimale Lösung gefunden werden. Das Gleiche gilt auch für Sprechkanülen bzw. -ventile für Laryngektomierte, die in ein Konzept zur ganzheitlichen Rehabilitation des kehlkopflosen Patienten einbezogen werden müssen - sowohl für die sprachliche Kommunikationsfähigkeit als auch für die Funktionsänderung des oberen Aerodigestivtrakts. 


\section{Epithesen, Tamponaden, Klebstoffe}

Rehabilitation in anderer Form, nämlich in Bezug auf Wiederherstellung des äußeren Erscheinungsbildes bei plastischchirurgisch nicht oder schwer behebbaren Defekten, wird durch künstliche Gesichtsteile, Epithesen, erreicht. Moderne Silikone und Fixierungen über knochenverankerte Magnete geben auch nach verstümmelnden Eingriffen dem Patienten ein Gesicht wieder, das ihn aus einer gesellschaftlichen Isolation befreit. Osseointegrierte Titanschrauben haben dabei ein großes Potenzial zur sicheren Fixierung äußerlich aufgesetzter Kunststoffteile und sonstiger Hilfsmittel. Im Referat wird ein Überblick über die Möglichkeiten der extraoralen Implantologie gegeben.

Im Gegensatz zu endonasalen Stents, z. B. zum Offenhalten des Zugangs zur Stirnhöhle, werden Nasentamponaden nur kurzfristig zur Blutstillung bzw. Sicherung eines operativen Ergebnisses eingesetzt und sind somit keine Implante. Andererseits kommen ihre Oberflächen mit frischen Gewebedefekten, z. B. nach endonasaler Nebenhöhlenchirurgie, in Berührung, sodass bestimmte Kautelen bezüglich Einsatz, Nutzen und Risiken berücksichtigt werden müssen. In der endonasalen Chirurgie sollten nach individuellem Abwägen der Notwendigkeit nur noch konventionelle Tamponaden mit glatter Oberfläche verwendet werden. Berücksichtigt werden nur moderne Nasentamponaden, die nicht nur als druckauslösende, raumfüllende Formkörper fungieren. Hämostatische bzw. resorbierbare gehen zwar in die gewünschte Richtung, tragen jedoch oft das Risiko vermehrter Synechien oder Fremdkörperreaktionen aufgrund einer Inkorporation in die Schleimhaut in sich. Einfaches Zukleben der Nase kann sehr effektiv ein feuchtes Milieu zur Optimierung der Wundheilung gewährleisten.

Letztlich zu den Biomaterialien gezählt werden können auch Gewebeklebstof$\mathrm{fe}, \mathrm{zu}$ denen umfangreiche Forschungen im Gange sind. Im HNO-Bereich müssen die spezifischen Anforderungen der unterschiedlichen Anwendungsgebiete wie beispielsweise im Schleimhautmilieu sowie im Bereich des Knochens, Knorpels und der peripheren Nerven berücksichtigt werden, außerdem Probleme unterschiedlicher mechanischer Belastung der Klebestellen. Darüber hinaus müssen im Sinne einer minimal-invasiven Chirurgie geeignete Applikationssysteme für den mikroskopischen und endoskopischen Einsatz entwickelt werden.

Alle genannten Themenkomplexe werden durch die Referenten zur Kongresseröffnung ausführlich vorgetragen.

\section{Wissenschaftlicher Vorgeschmack in diesem Heft}

Um jedoch auch die große wissenschaftliche Bandbreite der Tagung anzudeuten, habe ich einige zu Vorträgen oder zu Rundtischgesprächen eingeladene Experten geben, an Hand von in diesem Heft publizierten Artikeln einen Vorgeschmack auf Rostock im Mai 2009 zu geben. Dabei werden folgende Themenkreise berührt: Visionen zum Kochleaimplantat, Aktuelles zur Menière-Erkrankung, Innovationen zu „mikrobildgebenden Verfahren“, Neues zum Reflux bzw. aktuelle Konzepte der Onkologie.

\section{Einladung}

Rostock und die Ostseeküste im Mai - das verspricht nicht nur wissenschaftlichen Gedankenaustausch und Gewinn, sondern soll allen Kongressbesuchern auch das moderne, maritim geprägte Flair einer alten Hansestadt und ihrer schönen Umgebung nahe bringen.

Ich freue mich auf Ihr Kommen!

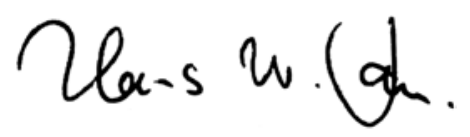

Ihr Hans Wilhelm Pau

\section{Korrespondenzadresse Prof. Dr. H.-W. Pau}

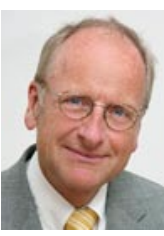

Universitäts-Hals-

Nasen-Ohren-Klinik

Doberaner Straße 137-139,

18057 Rostock

hans-wilhelm.pau@

med.uni-rostock.de

\section{Leitfaden zum Risikomanage- ment in Krankenhäusern ver- öffentlicht}

"Schutz Kritischer Infrastruktur: Risikomanagement im Krankenhaus" heißt ein neuer Leitfaden des Bundesamtes für Bevölkerungsschutz und Katastrophenhilfe (BBK), der dabei helfen soll, die Funktionsfähigkeit eines Krankenhauses in Krisensituationen sicher zu stellen. Um mögliche Gefährdungen in Krankenhäusern bereits im Vorfeld zu erkennen und Schäden durch vorbeugende Maßnahmen zu begrenzen, hat das BBK zusammen mit Medizinern, Geisteswissenschaftlern, Ingenieuren und Verwaltungsfachleuten Empfehlungen erarbeitet. Die kompakte, anwendungsorientierte Broschüre wendet sich an Entscheidungsträger und Sicherheitsfachleute in Krankenhäusern und enthält zahlreiche inhaltliche und methodische Hinweise, die den Anwender aktiv bei der Umsetzung eines Risikomanagement-Prozesses unterstützen. Eine CD-ROM mit weiteren Arbeitsmaterialien und Informationen liegt dem Leitfaden bei. Die Broschüre kann über die Seiten des BBK kostenfrei bezogen oder heruntergeladen werden.

Quelle:

Bundesamt für Bevölkerungsschutz und Katastrophenhilfe, Bonn www.bbk.bund.de 